Published in "International Journal of Antimicrobial Agents 55(2): 105802, 2020" which should be cited to refer to this work.

\title{
Occurrence of CTX-M-15- and MCR-1-producing Enterobacterales in pigs in Portugal: Evidence of direct links with antibiotic selective pressure
}

\author{
Claudine Fournier ${ }^{\mathrm{a}}$, Marta Aires-de-Sousa ${ }^{\mathrm{b}, \mathrm{c}}$, Patrice Nordmann ${ }^{\mathrm{a}, \mathrm{d}, \mathrm{e}, \mathrm{f}}$, Laurent Poirel ${ }^{\mathrm{a}, \mathrm{d}, \mathrm{e}, *}$ \\ ${ }^{a}$ Medical and Molecular Microbiology Unit, Faculty of Science and Medicine, University of Fribourg, Fribourg, Switzerland

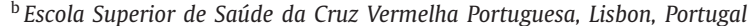 \\ ${ }^{c}$ Laboratory of Molecular Genetics, Instituto de Tecnologia Química e Biológica António Xavier, Universidade Nova de Lisboa, Oeiras, Portugal \\ ${ }^{\mathrm{d}}$ INSERM European Unit, University of Fribourg, Fribourg, Switzerland \\ e Swiss National Reference Centre for Emerging Antibiotic Resistance, University of Fribourg, Fribourg, Switzerland \\ ${ }^{\mathrm{f}}$ Institute for Microbiology, University of Lausanne and University Hospital Centre, Lausanne, Switzerland
}

\begin{abstract}
Aims: To undertake a prospective analysis of the occurrence of colistin-resistant and extended-spectrum ß-lactamase (ESBL)-producing Enterobacterales colonizing pigs at two farms in Portugal, and to evaluate the putative correlations with usage of different antibiotics.

Materials and methods: One hundred and two faecal samples recovered from two different Portuguese pig farms were screened for polymyxin-resistant and ESBL-positive Enterobacterales. The authors had undertaken a study at one of the farms previously, but the use of colistin has since been banned; zinc oxide and amoxicillin are used as prophylactic and curative drugs, respectively, at this farm. The other farm included in this study used zinc oxide alone.

Results: Ninety-three ESBL-producing isolates (62 Escherichia coli, 29 Klebsiella pneumoniae, one Enterobacter aerogenes and one Enterobacter cloacae) and 17 colistin-resistant isolates (12 E. coli, four K. pneumoniae and one E. cloacae) were recovered. Among the ESBL producers, the majority (84\%) produced CTX-M-15, while the others produced CTX-M-1 or CTX-M-9. Many different strain and plasmid backgrounds were identified, ruling out a massive dissemination of one major clone. In total, 17 colistin-resistant isolates were recovered, all from the first farm. All produced MCR-1, corresponding to 12 E. coli (10 clones) and three K. pneumoniae (two clones). The MCR-1 producers were all recovered from the farm where colistin had been used 2 years previously.

Conclusion: This study showed a surprisingly high rate of CTX-M-15 producers at two Portuguese pig farms. A link was found between antibiotic selective pressure (B-lactam or polymyxin) and the corresponding resistance rate.
\end{abstract}

\section{Introduction}

The worldwide increase in acquired antimicrobial resistance represents a problem for human and veterinary medicine. As a consequence, the current position to combat the rise in antibiotic resistance in humans includes the so-called 'One-Health approach' which considers the problem of resistance as a whole, including antibiotic use in humans and in animals [1].

\footnotetext{
* Corresponding author. Medical and Molecular Microbiology Unit, Department of Medicine, Faculty of Science, University of Fribourg, Chemin du Musée 18, CH-1700 Fribourg, Switzerland. Tel.: +41 263009582.

E-mail address: laurent.poirel@unifr.ch (L. Poirel).
}

Acquired resistance to broad-spectrum cephalosporins in Enterobacterales is mainly related to acquisition of extended-spectrum $\beta$-lactamase (ESBL) genes, particularly $b a_{\mathrm{CTX}-\mathrm{M}}$-like genes [2]. bla $_{\mathrm{CTX}-\mathrm{M}-15}$ is widely distributed in human isolates, including in the community, and bla $a_{\text {CTX-M-1 }}$ is the most commonly identified ESBL gene among animal isolates [3]. A large number of epidemiological studies have been conducted worldwide to evaluate whether animals might represent important sources of ESBL-producing isolates affecting humans $[3,4]$.

Colistin is used as an antimicrobial drug in veterinary medicine for purposes including enteric infections and prophylactic treatment, and as growth supplementation in livestock feed in some countries. There is concern about recent identification of plasmidencoded resistance to polymyxins since identification of the 
plasmid-borne mcr-1 gene in 2016 at a pig farm in China [5]. The MCR-1 enzyme, a phosphoethanolamine transferase conferring acquired resistance to polymyxins in Enterobacterales, has mainly been found in animal isolates. This enzyme modifies the lipopolysaccharide of the external membrane of Gram-negative bacteria by adding a phosphoethanolamine group to the lipid A moiety, therefore leading to resistance [6]. The mcr-1 gene has been epidemiologically related to environmental and animal samples, mostly in Escherichia coli from worldwide origin. Noteworthy, the $m c r-1$ gene has been widely identified among E. coli strains recovered from pigs [6,7].

Two years ago, the authors conducted a study to evaluate the occurrence of colistin-resistant enterobacterial isolates at two pig farms in Portugal (Farm 1 and Farm 2); a very high rate of MCR-1 producers (mainly E. coli isolates) was identified at both farms, accounting for $98 \%$ of the pigs [8]. Both farms were feeding the pigs with colistin-supplemented nutriments, and they also used zinc oxide and amoxicillin on a regular basis. A link between colistin selective pressure and the frequent occurrence of colistin-resistant isolates was hypothesized. The use of colistin has since been banned at Farm 2, with zinc oxide and amoxicillin being kept as prophylactic and curative drugs, respectively. The two farms followed the newly-implemented Portuguese National Action Plan for the Reduction of Antibiotic Use in Animals under the auspices of the European Centres for Disease Control and Prevention [https://ecdc.europa.eu/en/ publications-data/directory-guidance-prevention-and-control/ antimicrobial-resistance-strategies].

In order to better evaluate the link between colistin usage and colistin resistance, and to further our knowledge on the rate of colistin-resistant and ESBL-producing enterobacterial isolates among pigs in Portugal, the authors conducted another prospective study. Two pig farms were sampled, one of which was 'Farm 2' from the previous study, and a new farm (Farm 3). No antibiotics are given at Farm 3, and zinc oxide is given as prophylaxis. The objectives of this study were: (i) to evaluate the prevalence of ESBL-producing and polymyxin-resistant gene isolates at these pig farms; and (ii) to evaluate whether the colistin ban at Farm 2 may have resulted in a decrease in resistant isolates.

\section{Materials and methods}

\subsection{Sampling}

Rectal swabs were collected in June 2018 from pigs at two different farms, both located near Lisbon, Portugal but $>50 \mathrm{~km}$ apart. Swabs were analysed immediately after recovery.

\subsection{Screening of resistant isolates}

Each sample was enriched overnight in Luria-Bertani broth. One calibrated inoculated loop $(10 \mu \mathrm{L})$ of each sample was plated on to SuperPolymyxin selective agar medium that contains $3.5 \mu \mathrm{g} / \mathrm{mL}$ of colistin and $10 \mu \mathrm{g} / \mathrm{mL}$ of daptomycin (ELITechGroup, Signes, France) to select for colistin-resistant Gram-negative bacteria [9]. In addition, screening of ESBL-producing isolates was performed using ChromID ESBL (bioMérieux, La Balme les Grottes, France) selective agar medium, screening of pan-aminoglycoside-resistant Gram-negative bacteria was performed using SuperAminoglycoside selective medium [10], and screening of carbapenemase-producing Enterobacterales was performed using SuperCarba and CarbaSmart selective plates (bioMérieux) [11]. Antibiotic susceptibility testing was performed by disk diffusion assay, except for colistin which was tested by broth microdilution.

\subsection{Confirmation of resistance patterns}

Colistin-resistant and ESBL-producing isolates were tested by the Rapid Polymyxin NP test [12] and the Rapid ESBL NP test [13], respectively, in order to confirm their resistance patterns. For all selected isolates, identification at species level was performed using the API20E system (bioMérieux).

\subsection{Molecular assays}

Screening of ESBL-encoding genes (namely $b l a_{\mathrm{TEM}}$, bla $a_{\mathrm{SHV}}$ and bla $_{\text {CTX-M }}$ ) was performed by polymerase chain reaction (PCR) amplification using specific primers [14]. Similarly, screening of $\mathrm{mcr}$ genes (mcr-1 to -9 ) was performed using previously published primers [15]. Amplicons were further sequenced to identify the resistance gene precisely (Microsynth, Balgach, Switzerland).

\subsection{Plasmid characterization}

Plasmids bearing the $b a_{\mathrm{CTX}-\mathrm{M}}$ gene and the $m c r-1$ gene were analysed by performing conjugation assays, and extracted by the Kieser method followed by transformation. Azide-resistant E. coli J53 was used as a recipient for mating-out assays on filter, and TOP10 E. coli was used as a recipient for transformation [8]. All E. coli transformants and $E$. coli transconjugants were further checked by PCR to confirm the success of the experiment. PCR-based replicon typing was perfomed on both transconjugants and the transformants [16]. Transconjugants for ESBL-producing isolates were selected on Luria-Bertani agar plates supplemented with sodium azide $(100 \mu \mathrm{g} / \mathrm{mL})$ and cefotaxime $(1 \mu \mathrm{g} / \mathrm{mL})$. Transformants for ESBL-producing isolates were selected on Luria-Bertani agar plates supplemented with cefotaxime $(1 \mu \mathrm{g} / \mathrm{mL})$. Selection of MCR-1producing E. coli transconjugants was performed on Luria-Bertani agar plates supplemented with sodium azide $(100 \mu \mathrm{g} / \mathrm{mL})$ and colistin $(1.5 \mu \mathrm{g} / \mathrm{mL})$.

\subsection{Evaluation of clonality}

The clonal relationship of E. coli and K. pneumoniae isolates was evaluated by pulsed-field gel electrophoresis (PFGE) analysis and multi-locus sequence typing.

\section{Results}

\subsection{Occurrence of ESBL-producing and colistin-resistant Enterobacterales}

In total, 102 rectal swabs were collected [51 pigs from each farm (i.e. Farm 2 and Farm 3)]. Delivery of colistin-supplemented food was stopped soon after the authors' previous sampling at Farm 2, but amoxicillin and zinc oxide are still used (0.15\% supplementation in feed regimens). At Farm 3, zinc oxide (0.15\%) alone is used as antimicrobial treatment for the prevention of postweaning diarrhoea. The authors no longer had access to Farm 1.

Of the 51 samples collected from Farm 2, 64 ESBL-producing isolates and 14 colistin-resistant isolates were recovered. At Farm 3, 29 ESBL-producing isolates and three colistin-resistant isolates were isolated from the 51 rectal swabs. No acquired panaminoglycoside resistance or carbapenemase-producing Enterobacterales were recovered from the samples.

\subsection{Determination of ESBL resistance determinants}

Of the 93 ESBL-producing isolates, there were 62 E. coli, 29 Klebsiella pneumoniae, one Enterobacter cloacae and one Enterobacter aerogenes (Table 1$)$. Seventy-nine $(84 \%)$ isolates produced 
Table 1

Diversity and sequence types of extended-spectrum beta-lactamase (ESBL)- and MCR-1-producing isolates.

\begin{tabular}{|c|c|c|c|c|}
\hline Farm & $\begin{array}{l}\text { E. coli ST } \\
\text { - ESBL type }\end{array}$ & $\begin{array}{l}\text { K. pneumoniae ST } \\
\text { - ESBL type }\end{array}$ & $\begin{array}{l}\text { E. coli ST } \\
\text { MCR-1 }\end{array}$ & $\begin{array}{l}\text { K. pneumoniae ST } \\
\text { MCR-1 }\end{array}$ \\
\hline 2 & $\begin{array}{l}\text { ST10 - CTX-M-9 }(n=4) \\
\text { ST44 -CTX-M-15 }(n=1) \\
\text { ST410 - CTX-M-1 }(n=2) \\
\text { ST410 - CTX-M-9 }(n=1) \\
\text { ST410 - CTX-M-15 }(n=20) \\
\text { ST480 - CTX-M-15 }(n=1) \\
\text { ST1665 - CTX-M-15 }(n=1) \\
\text { ST2437 - CTX-M-15 }(n=2) \\
\text { ST2496 - CTX-M-1 }(n=1) \\
\text { ST2496 - CTX-M-15 }(n=1) \\
\text { ST3014 - CTX-M-15 }(n=1)\end{array}$ & $\begin{array}{l}\text { ST1427 - CTX-M-1 }(n=3) \\
\text { ST1427 - CTX-M-15 }(n=25)\end{array}$ & $\begin{array}{l}\text { ST10 }(n=4) \\
\text { ST410 }(n=2) \\
\text { ST1406 }(n=3)\end{array}$ & $\begin{array}{l}\text { ST1427 }(n=2) \\
\text { ST1563 }(n=1)\end{array}$ \\
\hline 3 & $\begin{array}{l}\text { ST10 - CTX-M-15 }(n=2) \\
\text { ST58 - CTX-M-15 }(n=1) \\
\text { ST314 - CTX-M-1 }(n=2) \\
\text { ST314 - CTX-M-15 }(n=1) \\
\text { ST2473 - CTX-M-1 }(n=1) \\
\text { ST2526 - CTX-M-15 }(n=19) \\
\text { ST7586 - CTX-M-15 }(n=1)\end{array}$ & ST896 - CTX-M-1 $(n=1)$ & $\begin{array}{l}\text { Unknown ST }(n=1) \\
\text { ST2290 }(n=2)\end{array}$ & \\
\hline
\end{tabular}

E. coli, Escherichia coli; K. pneumoniae, Klebsiella pneumoniae; ST, sequence type.

CTX-M-15 (51 E. coli, 26 K. pneumoniae, one E. cloacae and one E. aerogenes), 10 isolates produced CTX-M-1 (six E. coli and four $K$. pneumoniae) and five isolates produced CTX-M-9 (all E. coli). All ESBL-producing $K$. pneumoniae isolates except four produced the CTX-M-15 enzyme, all recovered from a single farm, and belonged to sequence type (ST) 1427 (see below).

At Farm 2, all ESBL producers were found to be resistant to chloramphenicol, $96 \%$ were resistant to sulfamethoxazole/trimethoprim, 92\% were resistant to tetracycline, and $74 \%$ were resistant to kanamycin, tobramycin and sulfonamides. At Farm 3, co-resistances identified were much lower, with only $44 \%$ of ESBL producers resistant to tetracycline and sulfonamides, $22 \%$ resistant to sulfamethoxazole/trimethoprim, and $11 \%$ resistant to chloramphenicol, tobramycin and kanamycin.

\subsection{Clonal relationship}

Among the 35 different ESBL-producing E. coli isolates recovered from Farm 2, 21 PFGE patterns were identified, corresponding to eight different STs (ST10, ST44, ST410, ST480, ST1665, ST2437, ST2496, ST3014) (Table 1). Among the 27 ESBL-producing E. coli isolates recovered from Farm 3, 10 PFGE patterns were identified, corresponding to six different STs (ST10, ST58, ST314, ST2473, ST2526, ST7586). ST10 was therefore the only common strain background identified in both farms.

\subsection{Determination of colistin resistance determinants}

Of the 17 colistin-resistant isolates, $12 \mathrm{E}$. coli, four $\mathrm{K}$. pneumoniae and one E. cloacae were recovered. Sixteen of the 17 colistinresistant isolates possessed the $\mathrm{mcr}-1$ gene, corresponding to $12 \mathrm{E}$. coli belonging to 10 clonal backgrounds, three K. pneumoniae belonging to two clonal backgrounds, and one E. cloacae isolate. One colistin-resistant $K$. pneumoniae isolate was negative for all $\mathrm{mcr}$ genes but possessed a chromosomal modification known to lead to colistin resistance, namely a truncation of the $\operatorname{mgrB}$ gene [6]. Nine of the 12 MCR-1-producing E. coli, all the MCR-1 K. pneumoniae and the MCR-1-producing E. cloacae were recovered from Farm 2. The nine E. coli clones belonged to three different STs: ST10, ST1406 and ST410. The three mor-1-positive K. pneumoniae and the isolate exhibiting chromosomally-encoded colistin resistance all belonged to ST1427.
Three isolates co-produced both an ESBL and MCR-1: one

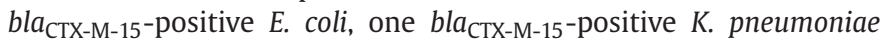
and one bla $a_{\mathrm{CTX}-\mathrm{M}-1}$-positive $K$. pneumoniae. All MCR-1 producers were resistant to tobramycin, $73 \%$ were resistant to amoxicillin, $36 \%$ were resistant to tetracycline and chloramphenicol, and $27 \%$ were resistant to sulfonamides.

\subsection{Plasmid analysis}

Analysis of plasmids encoding the ESBL determinants showed that, in most cases $(52 \%)$, the $b a_{\mathrm{CTX}-\mathrm{M}-15}$ gene was carried by a conjugative IncFIA/FIB plasmid encoding co-resistance to tetracycline, kanamycin, tobramycin, sulfonamides and trimetho-

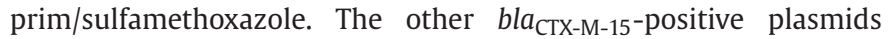
identified belonged to FIA, FIB, HI1, I1, P and FIC incompatibility groups. The mcr-1 gene was carried by a self-conjugative IncHI2 plasmid.

\section{Discussion}

The rates of ESBL- and MCR-1-producing enterobacterial isolates identified in this study were $91 \%$ and $17 \%$, respectively. However, a significant discrepancy was evidenced, as the ESBL rates were $86 \%$ and $55 \%$ for Farms 2 and 3, respectively. This correlates with the use of a ß-lactam at Farm 2. Likewise, the rates of colistin-resistant isolates were 27\% and 6\% for Farms 2 and 3, respectively. This correlates with previous use of colistin at Farm 2, which ceased 2 years ago. The rate of colistin-resistant isolates was 98\% at Farm 2 in 2016, so the current results represent a highly significant decrease. With such follow-up at the same farm and with the exact same methodology, this shows the significant benefit of banning the use of colistin in terms of decreased prevalence of the level of colistin resistance.

However, the high rate of ESBL producers found at Farm 2 indicates that significant efforts are still needed to reduce the use of ß-lactams; unfortunately, the authors were unable to collect precise data on amoxicillin consumption at Farm 2, as it was given on a non-regular basis to treat cases of diarrhoea in pigs.

Evaluation of clonal diversity among ESBL-producing E. coli identified 21 clones for Farm 2 and 10 clones for Farm 3, whereas seven and one clones, respectively, were identified among the ESBL-producing $K$. pneumoniae isolates. This shows that, in line with previous observations with colistin-resistant isolates, the high 
resistance rates observed are not due to circulation of one or a few specific clones, but rather by a high diversity of clonal backgrounds in which some epidemic plasmids might be acquired.

E. coli isolates belonging to ST10, ST58, ST314, ST1665, ST2526 and ST3014 have been identified previously in animal faecal samples, and E. coli ST10 and ST410 have been reported in humans [17]. This shows that the E. coli STs identified in this study might be very widespread, and not specifically considered as animal commensals, further highlighting their potential to spread.

Among the K. pneumoniae STs found in this study, the identification of ST1427 is noteworthy as the same clonal background harbouring the bla $a_{\mathrm{CTX}-\mathrm{M}-15}$ ESBL gene was responsible for a nosocomial outbreak in the Netherlands in 2015 [18].

All the colistin-resistant isolates identified in this study possessed the mcr-1 gene, which was therefore the main colistin resistance determinant. The $m c r-1$ gene was always identified on IncHI2-type plasmids, which is in accordance with previous findings showing that the IncHI2 plasmid was the most common plasmid support of mcr-1 (54\%) among E. coli and K. pneumoniae isolates recovered in Portugal [8].

All isolates possessed the ESBL encoding gene and the mcr-1 gene on distinct plasmids, with no co-localization being observed.

This study reports high rates of ESBL and MCR-1 production among enterobacterial isolates recovered from farmed pigs in Portugal, colonizing $75 \%$ and $11 \%$ of the animals, respectively. The rate of ESBL producers was very high at Farm 2 where amoxicillin was used, albeit sporadically; $100 \%$ of the screened animals were carriers of at least one ESBL-producing isolate. Conversely, this rate was lower (57\%) at Farm 3 where no ß-lactam antibiotics are given. Surprisingly, 63 of the 76 ESBL producers harboured the $b l a_{\text {CTX-M-15 }}$ gene. This ESBL gene is notoriously and widely disseminated among human E. coli, not only in nosocomial settings but also in the community. Such a high rate of CTX-M-15 producers was therefore not expected, and possible human faecal contamination could be hypothesized. Water samples were subsequently taken from the tank supplying water to the animals at Farm 2, along with soil samples from the same farm, but neither ESBL- nor MCR-1-producing E. coli isolates were recovered (data not shown). Therefore, this phenomenon remains unexplained, and future investigations will be conducted to identify a potential source of contamination by CTX-M-15 producers.

This study found highly significant discrepancies in the prevalence of MCR-1-producing E. coli between the two farms. When considering the 51 pigs treated with zinc oxide alone (Farm 3), only three E. coli isolates harbouring $m c r-1$ were recovered (5.9\%). Conversely, 14 (27.4\%) MCR-1-producing isolates were recovered from the farm where colistin had been widely used until 2 years previously (Farm 2). Nevertheless, this rate was much lower than that found at Farm 2 in the authors' previous study (98\%) [8]. It could be speculated that this decrease was likely due to a lack of selective pressure with colistin. This observation is encouraging, as it demonstrates that the colistin ban had a highly significant positive outcome with respect to bacterial ecology, as expected. Interesting and positive outcomes in terms of control and decrease of antimicrobial resistance have also been reported recently from China (where colistin has been banned for veterinary use since November 2015). Li et al. [19] reported a drastic decrease in the rate of colistin resistance in E. coli in healthy pigs. Continuous efforts are therefore required to inform and evaluate the options that might reduce antibiotic resistance overall, including that to lastresort drugs such as colistin.

Funding: This work was supported, in part, by the University of Fribourg, the Swiss National Science Foundation (Projects FNS40AR40_173686 and FNS-407240_177381), INSERM, Fundação para a Ciência e a Tecnologia (Project PTDC/DTP-EPI/0842/2014), and by Project LISBOA-01-0145-FEDER-007660 (Microbiologia Molecular, Estrutural e Celular) funded by FEDER funds through COMPETE2020 - Programa Operacional Competitividade e Internacionalização.

Competing interests: None declared.

Ethical approval: Not required.

\section{References}

[1] Calistri P, Iannetti S, Danzetta ML, Narcisi V, Cito F, Sabatino DD, et al. The components of 'One World - One Health' approach. Transbound Emerg Dis 2013;60(Suppl. 2):4-13.

[2] Rossolini GM, D’Andrea MM, Mugnaioli C. The spread of CTX-M-type extended-spectrum ß-lactamases. Clin Microbiol Infect 2008;14(Suppl. 1):33-41.

[3] Madec JY, Haenni M, Nordmann P, Poirel L. Extended spectrum ß-lactamase/AmpC and carbapenemase producing Enterobacteriaceae in animals: a threat for humans? Clin Microbiol Infect 2017;23:826-33.

[4] Poirel L, Madec JY, Lupo A, Schink AK, Kieffer N, Nordmann P, et al. Antimicrobial resistance in Escherichia coli. Microbiol Spectr 2018;6.

[5] Liu YY, Wang Y, Walsh TR, Yi LX, Zhang R, Spencer J, et al. Emergence of plasmid-mediated colistin resistance mechanism MCR- 1 in animals and human beings in China: a microbiological and molecular biological study. Lancet Infect Dis 2016;16:161-8.

[6] Poirel L, Jayol A, Nordmann P. Polymyxins: antibacterial activity, susceptibility testing, and resistance mechanisms encoded by plasmids or chromosomes. Clin Microbiol Rev 2017;30:557-96.

[7] Baron S, Hadjadj L, Rolain JM, Olaitan AO. Molecular mechanisms of polymyxin resistance: knowns and unknowns. Int J Antimicrob Agents 2016;48:583-91.

[8] Kieffer N, Aires-de-Sousa M, Nordmann P, Poirel L. High rate of MCR-1-producing Escherichia coli and Klebsiella pneumoniae among pigs, Portugal. Emerg Infect Dis 2017;23:2023-9.

[9] Nordmann P, Jayol A, Poirel L. A universal culture medium for screening polymyxin-resistant Gram-negative isolates. J Clin Microbiol 2016;54:1395-9.

[10] Nordmann P, Mazé A, Culebras E, Dobias J, Jayol A, Poirel L. A culture medium for screening $16 \mathrm{~S}$ rRNA methylase-producing pan-aminoglycoside resistant Gram-negative bacteria. Diagn Microbiol Infect Dis 2018;91:118-22.

[11] Nordmann P, Girlich D, Poirel L. Detection of carbapenemase producers in Enterobacteriaceae by use of a novel screening medium. J Clin Microbiol 2012;50:2761-6.

[12] Nordmann P, Jayol A, Poirel L. Rapid detection of polymyxin resistance in Enterobacteriaceae. Emerg Infect Dis 2016;22:1038-43.

[13] Nordmann P, Dortet L, Poirel L. Rapid detection of extended-spectrum- $\beta$-lactamase-producing Enterobacteriaceae. J Clin Microbiol 2012;50:3016-22

[14] Poirel L, Dortet L, Bernabeu S, Nordmann P. Genetic features of bla $a_{\mathrm{NDM}-1}$-positive Enterobacteriaceae. Antimicrob Agents Chemother 2011:55:5403-7.

[15] Kieffer N, Royer G, Decousser JW, Bourrel AS, Palmieri M, Ortiz De La Rosa JM, et al. mcr-9, an inducible gene encoding an acquired phosphoethanolamine transferase in Escherichia coli, and its origin. Antimicrob Agents Chemother 2019 pii:AAC.00965-19.

[16] Carattoli A, Bertini A, Villa L, Falbo V, Hopkins KL, Threlfall EJ. Identification of plasmids by PCR-based replicon typing. J Microbiol Methods 2005;63:219-28.

[17] Lopez-Cerero L, Egea P, Serrano L, Navarro D, Mora A, Blanco J, et al. Characterisation of clinical and food animal Escherichia coli isolates producing CTX-M-15 extended-spectrum ß-lactamases to ST410 phylogroup A. Int J Antimicrob Agents 2011;37:365-7.

[18] Zhou K, Lokate M, Deurenberg RH, Arends J, Lo-Ten Foe J, Grundmann H, et al. Characterization of a CTX-M-15-producing Klebsiella pneumoniae outbreak strain assigned to a novel sequence type (1427). Front Microbiol 2015;6:1250.

[19] Li W, Hou M, Liu C, Xiong W, Zeng Z. Dramatic decrease in colistin resistance in Escherichia coli from a typical pig farm following restriction of colistin use in China. Int J Antimicrob Agents 2019;53:707-70. 